\title{
The inflammatory reflex and neural tourniquet: harnessing the healing power of the vagus nerve
}

\author{
Jared M Huston ${ }^{*}, 1$ \& Jason R Fritz ${ }^{2}$ \\ ${ }^{1}$ Assistant Professor of Surgery \& Science Education, Division of Trauma \& Acute Care Surgery, Donald and Barbara Zucker School \\ of Medicine at Hofstra/Northwell, Assistant Professor, Center for Bioelectronic Medicine, The Feinstein Institute for Medical \\ Research, 300 Community Drive, Manhasset, NY 11030, USA \\ ${ }^{2}$ Staff Scientist, Center for Bioelectronic Medicine, The Feinstein Institute for Medical Research, 300 Community Drive, Manhasset, \\ NY 11030, USA \\ * Author for correspondence: Tel.: +1 516233 3610; JHuston@northwell.edu
}

The CNS helps protect against tissue injury. The most important priorities include limiting blood loss and systemic inflammation. We elucidated two endogenous neural pathways that rapidly and specifically improve hemostasis and decrease inflammation through vagus nerve signaling. Activation of the neural tourniquet or inflammatory reflex via electrical vagus nerve stimulation (VNS) significantly improves outcomes in preclinical disease models. Currently, VNS is clinically approved for the treatment of medically refractory epilepsy and depression. The growing field of bioelectronic medicine will help physicians harness the Neural Tourniquet ${ }^{\mathrm{TM}}$ and inflammatory reflex for clinical use as well. Considering the substantial harm caused by uncontrolled bleeding and inflammation, electrical VNS may dramatically improve the care of millions of patients.

First draft submitted: 11 August 2017; Accepted for publication: 12 October 2017; Published online: 14 December 2017

Keywords: bioelectronic medicine $\bullet$ bleeding $\bullet$ hemorrhage $\bullet$ neural tourniquet $\bullet$ vagus nerve stimulation

Advances in modern medicine have substantially improved outcomes for many diseases. Patients now expect to live longer and healthier lives thanks to improvements in disease diagnosis, treatment and prevention. Tantamount to this success are the enormous benefits of pharmaceuticals. For example, broad-spectrum antibiotics eliminate potentially lethal bacterial infections. Antirejection agents keep organ transplants functioning. Powerful chemotherapeutic agents shrink cancerous tumors.

While one cannot underestimate their importance, pharmaceuticals contribute to significant morbidity and mortality as well. A primary drawback is the lack of specificity for their intended target. Contributing to this problem are limitations on routes of administration. Most drugs are given orally or intravenously and then must circulate throughout the body before reaching the target. So while chemotherapy can kill cancer cells, systemic administration damages critical immune cells, leaving patients susceptible to opportunistic infections. Pharmaceuticals are also difficult to titrate appropriately and reverse their effects. Even newer, more efficacious oral anticoagulants lack effective antidotes that may increase the risk of bleeding following injury [1].

Overprescribing of pharmaceuticals is a serious concern. Once powerful antibiotics face increasing bacterial resistance, resulting in treatment failures, prolonged hospitalizations and greatly increased costs [2]. Another frightening example is the current opioid epidemic in the USA. Initiatives to better alleviate chronic pain may have resulted in significant numbers of patients developing dependency on narcotic pain relievers [3]. Maintaining patient compliance with prescription drugs is problematic as well. Dosing errors result in serious and potentially life threatening complications [4]. Finally, there is the monetary cost. Regulatory approval requires years of testing and millions of dollars in research and development costs, which are ultimately passed on to patients. Those who cannot afford these lifesaving treatments risk acquiring substantial medical debt or even falling into bankruptcy [5].

The challenge ahead is to develop drugs with greater specificity, better compliance, fewer side effects and lower costs. Rather than pursuing novel drug options, however, one can advocate for an entirely different approach. 
This new strategy is highly specific because the therapy is released within micrometers from the intended target. This therapy is safe because it endured thousands of years of testing and modifications. This method can increase compliance because patients may not have to remember to administer it every few hours. This treatment costs less because development occurred in nature's laboratory. This class of 'drugs' is neurotransmitters, and the way to harness them is through bioelectronic medicine.

\section{Bioelectronic medicine}

Bioelectronic medicine utilizes the nervous system to treat injury and disease [6]. The focus is on healing the body from within, rather than through administration of exogenous drugs. The mammalian nervous system has evolved to monitor and regulate the internal environment through highly sophisticated circuitry involving every major organ in the body [7]. Communication within this neural network occurs via electrical signals that travel rapidly and specifically along nerves. Upon reaching their intended cellular and molecular targets, these electrical signals cause the release of neurotransmitters. Through targeted manipulation of these neural signals, bioelectronic medicine harnesses endogenous pathways for therapeutic purposes. This is accomplished with nerve-stimulating or nerve-blocking devices that are either surgically implanted internally or applied externally to the skin. This noninvasive approach is particularly advantageous as it is administered more easily, tolerated better and costs less than traditional pharmacological agents. Through the speed and precision of neural signaling, bioelectronic medicine has the capacity to fundamentally change how we treat diseases while avoiding the side effects and other limitations of pharmaceuticals.

\section{Current indications}

Epilepsy is a seizure disorder characterized by abnormal nerve cell activity in the brain cortex. Approximately 70 million individuals are affected worldwide [8]. The underling cause in the majority of cases remains unknown. Multiple factors contribute to the increased risk of death in epilepsy patients, including uncontrollable seizures, the underlying cause of the disease, trauma and suicide [9]. With appropriate anticonvulsant medications, seizures can be controlled in a large majority of patients. Most of these drugs have mild side effects, although serious problems including bone marrow suppression, liver toxicity and birth defects are possible [10].

Depression is one of the most common psychiatric disorders, with approximately 20 million people affected annually in the USA alone. This serious mood disorder results from biologic, genetic and environmental factors. Interestingly, neural circuit function plays an important role in normal mood regulation and the development of depression [11]. The mainstay of treatment is typically a combination of psychotherapy and antidepressant pharmaceuticals. While these medications help millions of individuals, they are not without serious and potentially life threatening side effects.

Even after administration of multiple classes of drugs, some patients with epilepsy and depression do not derive benefits from anticonvulsants or antidepressants. Fortunately for these individuals, bioelectronic medicine offers another option. Since 1997, more than 100,000 individuals with medically refractory epilepsy or depression have benefited from this approach [12]. Electrical stimulation of the cervical vagus nerve stimulation (VNS) is accomplished using a surgically implantable pulse generator. The programmable stimulation parameters include frequency, amplitude, pulse width and on and off times. Multiple follow-up studies show that VNS significantly reduces symptoms of epilepsy and depression, even in those who fail all traditional medications $[12,13]$. Moreover, VNS is well tolerated and has a favorable side effect profile [14].

More recently, noninvasive bioelectronic medicine approaches have shown efficacy as well [15]. Transcutaneous electrical stimulation of the auricular branch of the vagus nerve innervating the cymba conchae of the ear can mimic invasive VNS and improve symptoms [16]. This cutaneous sensory nerve projects back to the nucleus of the solitary tract in the brainstem and activates the vagus nerve. Currently, this therapy is only approved in Europe, where patients can self-administer their treatment at home. Direct, transcutaneous electrical stimulation of the cervical vagus nerve is also feasible and has shown significant benefits [17]. Long-term studies will determine if patients can maintain adequate compliance with these noninvasive approaches, especially when treating chronic inflammatory diseases.

\section{Future indications}

The vagus nerve is the longest of the 12 cranial nerves and innervates major organs in the thoracic and abdominal cavities. The vagus nerve is the primary neural conduit for the parasympathetic arm of the autonomic nervous 


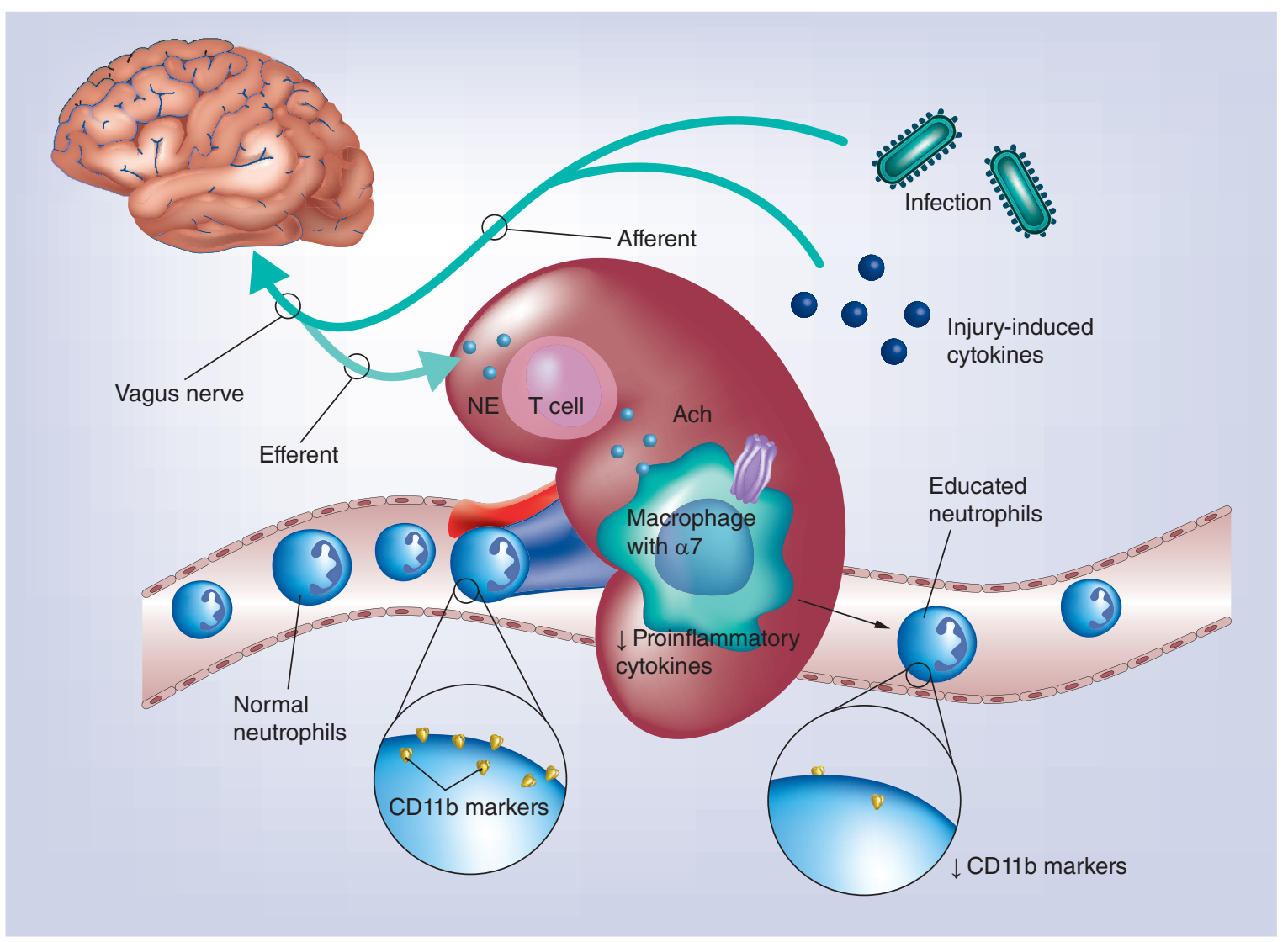

Figure 1. The inflammatory reflex.

Afferent vagus nerve signaling informs the brain about changes in peripheral inflammation and infection. Efferent vagus nerve signaling can inhibit systemic pro-inflammatory cytokine production and leukocyte trafficking at sites of tissue injury, inflammation and infection. This cholinergic anti-inflammatory pathway requires specific organ, cellular and molecular targets, including the spleen, acetylcholine-producing $T$ lymphocytes and the $\alpha 7$ nicotinic acetylcholine receptor subunit on splenic macrophages.

system. Functions under vagal control include heart rate, respiratory rate and gastrointestinal peristalsis. The vagus also innervates select skeletal muscles and branches such as the recurrent laryngeal nerve help control speech. Approximately $80-90 \%$ of vagal fibers are afferent in nature, and relay vital organ function status to the brain.

In addition to these critical functions, it is now known that the vagus comprises part of the neuro-immune axis by facilitating communication between the nervous and immune systems. For example, injection of IL-1 into the peritoneal cavity of rodents elicits a fever response from the brain [18]. Animals subjected to subdiaphragmatic vagotomy do not manifest a fever response, indicating that this response requires an intact vagus nerve [19]. Higher doses of cytokines, however, bypass this vagal pathway and elicit fever in vagotomized animals through entry at circumventricular organs [20]. Therefore, highly sensitive afferent vagal fibers appear positioned to rapidly detect changes in systemic inflammation.

Changes in peripheral inflammation relayed to the brain by the vagus nerve can activate the hypothalamicpituitary-adrenal axis, which releases cortisol and other stress hormones into the circulation [21]. The brain can also respond by increasing efferent vagus nerve firing. This combination of afferent and efferent vagus nerve signaling comprises the inflammatory reflex [22]. The efferent arm of the inflammatory reflex is termed the cholinergic anti-inflammatory pathway (CAP) because acetylcholine $(\mathrm{ACh})$ is the primary neurotransmitter of the vagus nerve [22]. For the past 20 years, our laboratory and others have investigated how the inflammatory reflex and the CAP monitor and regulate systemic inflammation (Figure 1) [23,24]. Activation of the CAP via either VNS or administration of a pharmacological cholinergic agonist limits proinflammatory cytokine production, inhibits apoptosis of critical immune cells, limits vital organ damage and improves survival in multiple models of systemic 
inflammation and sepsis, including collagen-induced arthritis, burns, colitis, myocardial ischemia, pancreatitis, stroke and polymicrobial abdominal sepsis [25-31] .

Electrical stimulation of the cervical vagus nerve in rodents significantly reduces systemic and organ specific proinflammatory cytokine production during endotoxic shock, with the greatest reduction coming in the spleen [32,33]. Splenectomy abolishes the cytokine-suppressing effects of VNS, indicating that the spleen is essential to the pathway [33]. Elucidation of this neural circuit is important because autonomic input to the spleen is through the splenic nerve, which releases norepinephrine rather than ACh. Studies show that cervical VNS releases norepinephrine in the spleen, and splenic neurectomy abolishes these effects [34]. This observation suggests that the vagus synapses with the splenic nerve in the celiac ganglion before reaching the spleen. Elevated NE concentrations in the spleen modulate specific T lymphocytes containing ChAT [35]. Genetic knockout of these cells abolishes anti-inflammatory vagus nerve signaling [35]. ACh release from ChAT $+\mathrm{T}$ cells downregulates cytokine-producing splenic macrophages through $\alpha 7$ nicotinic acetylcholine receptor subunits $(\alpha 7 \mathrm{nAChR})$ on the cell surface [35,36]. Electrical VNS or pharmacological cholinergic agonists fail to suppress inflammation in $\alpha 7$-deficient mice, and these animals have higher circulating cytokine concentrations following endotoxin challenge [36], suggesting that endogenous Inflammatory Reflex activity regulates systemic inflammation.

Based on years of successful preclinical work, there are now multiple clinical studies exploring the beneficial effects of electrical VNS. One recent study investigated whether electrical VNS using an implantable pulse generator can reduce clinical, biological and endoscopic markers of disease severity in seven patients with moderate to severe Crohn's disease despite corticosteroid and/or other immunosuppressive therapy [37]. VNS was used as an alternative to anti-TNF therapy or in treatment-naive patients. Stimulation parameters included an intensity of $0.5-1.5 \mathrm{~mA}$, frequency of $10 \mathrm{~Hz}$, pulse width of $500 \mu \mathrm{s}$, on time of 30 seconds and off time of 5 min. After a 6-month follow-up, the authors reported that five of seven patients had responded to VNS while two patients worsened and were removed from the study. Improvements were seen in the Crohn's disease activity index, C-reactive protein (CRP) and fecal calprotectin levels, and the Crohn's disease endoscopic index of severity. VNS therapy was also well tolerated and no major side effects were reported. Five of the seven subjects showed significant improvements, and no serious side effects were reported. VNS also restored sympatho-vagal balance as measured by heart rate variability in all patients.

Another recent pilot study investigated whether electrical VNS improves disease activity scores and reduces inflammatory makers in rheumatoid arthritis [38]. Seventeen patients with active RA despite pharmaceutical therapy with methotrexate underwent surgical implantation of an electrical pulse generator. Half of these patients had also failed previous treatment with at least two biological agents. Subjects received electrical stimulation once daily $(0.25-2.0 \mathrm{~mA}, 10 \mathrm{~Hz}, 250 \mu \mathrm{s}, 60 \mathrm{~s})$ on study day 0 and then again on study days 7 through 28 . At that point, any subjects who had not achieved a moderate or good clinical response had their stimulation frequency increased to four-times daily until study day 42. Stimulation resumed on day 56 until the end of the study on day 84 .

The authors reported a significant reduction in TNF production in cultured peripheral blood on day 42 compared with baseline. They also observed that TNF production increased significantly when the stimulator was turned off between days 42 and 56, but then decreased significantly again when stimulation resumed for the last 4 weeks of the study. In terms of signs and symptoms, the authors reported improved scores on a standard disease activity composite score (DAS28-CRP) when subjects were receiving VNS, and worsening of scores when stimulation was held. There were no serious adverse events, infections, withdrawals or deaths in the study.

To date, clinical studies of VNS have focused on diseases where pharmaceuticals have failed. While initially envisioned as a replacement for traditional drug therapies, bioelectronic medicine may also provide cures for diseases or disorders that currently have no pharmaceutical options. One such entity is the problem of bleeding. There are an estimated 100,000,000 inpatient and outpatient surgical procedures performed annually in the USA, with many millions more performed globally (CDC, National Center for Health Statistics). Arguably all surgeries have an inherent risk of bleeding. In the overwhelming majority of cases, patients do not receive prophylactic medications to reduce the incidence of bleeding. One notable exception is with joint replacement procedures, where administration of tranexamic acid has been shown to reduce bleeding severity [39,40]. Moreover, individuals who have acquired coagulopathies or other bleeding diatheses secondary to medications may receive treatment as well. For example, individuals taking warfarin or aspirin can receive plasma concentrates or platelets, respectively, to normalize hemostatic function.

In comparison to the risk of bleeding, multiple steps are taken to limit other common complications from surgical procedures. The most common site of infection following surgery occurs at the incision site. Multiple 
studies have shown that timely administration of pre-operative antibiotics significantly decreases rates of perioperative surgical site infection $[41,42]$. Postoperative pain also complicates surgical procedures. Along with their anesthesiology colleagues, surgeons can preemptively reduce pain incidence through the use of local anesthetics and nerve blocks. Venous thromboembolism is another frequent surgical complication. Studies dating back to the 1970s demonstrate that administration of heparin significantly reduces the incidence of both deep venous thrombosis and pulmonary embolism [43,44].

Even more problematic is when tissue trauma and hemorrhage is unpredictable. Traumatic injury is the third leading cause of death in the USA (CDC). The most common preventable cause of death following trauma is uncontrolled bleeding (CDC). Similar to these civilian statistics, trauma deaths in the military are also disproportionally caused by uncontrolled bleeding [45]. Advances in fluid and blood product resuscitation are helping to improve overall outcomes from massive hemorrhage [45]. Nevertheless, noncompressible hemorrhage from penetrating torso injuries remains the most common preventable cause of death in this arena [45].

The obvious challenge is that with a known risk of bleeding from surgery or trauma, we should administer prophylactic therapies to reduce this risk. If soldiers must enter dangerous combat situations, we should mitigate the bleeding risk and protect them before tragedy strikes. Moreover, any invasive medical procedure can carry a risk of bleeding, including tissue biopsies, endoscopies, colonoscopies, cardiac catheterizations and other interventional procedures. The overarching goal is to reduce the risk of hemorrhagic complications following surgery, invasive procedures and trauma by augmenting clotting function in healthy individuals.

\section{The Neural Tourniquet ${ }^{\mathrm{TM}}$}

Our studies of the inflammatory reflex reinforced that a highly regulated immune response is critical to survival. Regardless, uncontrolled inflammation is not the most immediate threat to life after injury. The most important priority is achieving hemostasis. Uncontrolled bleeding from a major blood vessel will kill the host much earlier than a dysregulated pro-inflammatory cytokine response. Due to the importance of monitoring and achieving hemostasis rapidly, we reasoned that the body would utilize the nervous system to accomplish this goal. Theoretically, a neuralbased clotting pathway can detect and modulate target organs and cells quickly and specifically, resulting in effective hemostasis and avoidance of potentially dangerous inadvertent clot formation elsewhere.

More than a decade ago, we set out to explore whether the nervous system modulates bleeding via the vagus nerve. We first developed an ear laceration and hemorrhage model in swine, a commonly utilized species to study bleeding [46]. 2- to 5-month-old male and female pigs weighing 25-30 kg received general anesthesia and then underwent a standardized ear laceration of $3 \mathrm{~cm}$ wide and $1.5 \mathrm{~cm}$ long with a scalpel. Duration of bleeding and total blood loss were recorded while animals were allowed to bleed freely from the wounds. Animals then received sham stimulation or electrical stimulation $(3.5 \mathrm{~mA}, 5 \mathrm{~Hz}, 2 \mathrm{~ms}, 30 \mathrm{~s})$ of the left cervical vagus nerve every 5 min for $30 \mathrm{~min}$. Ten minutes later, the contralateral ear was injured and identical measurements of bleeding time and blood loss were performed. Compared with baseline measurements, electrical VNS significantly shortened bleeding times by approximately $40 \%$. Moreover, VNS significantly reduced the total amount of blood loss by nearly $50 \%$. When compared with baseline values, sham VNS failed to decrease total bleeding time and blood loss.

To explain these improvements in hemostasis, hemodynamic parameters and measures of circulating cells and clotting function in systemic blood and shed blood from the injury site were analyzed via thromboelastography, platelet aggregometry and ELISA for thrombin-antithrombin (TAT) complexes [46]. Despite an initial concern for vagal parasympathetic effects on the heart, electrical VNS had no appreciable effect on either heart rate or blood pressure. Animals that received electrical VNS had significantly higher red blood cell counts and hematocrit levels compared with sham stimulation. The clinical significance of this finding is unclear, as the total overall blood loss in this hemorrhage model is relatively minor.

In terms of platelet clotting function, there were no differences in platelet aggregation between electrical or sham VNS. Using rotational thromboelastography, we observed that electrical VNS significantly reduces reaction (r) time of systemic blood compared with baseline values [46]. Thrombin generation studies revealed that compared with baseline values, blood from the injury site has significantly higher TAT concentrations in vagus nerve stimulated animals versus sham-stimulated animals [46]. This local difference in TAT concentration was statistically significant at $3 \mathrm{~min}$ after injury, whereas at $6 \mathrm{~min}$ the effect was less evident. There were no differences in circulating concentrations of TAT complexes between animals receiving electrical VNS or sham stimulation. Taken together, these findings suggest that accelerated clot formation specifically at the site of injury may account for improvements 


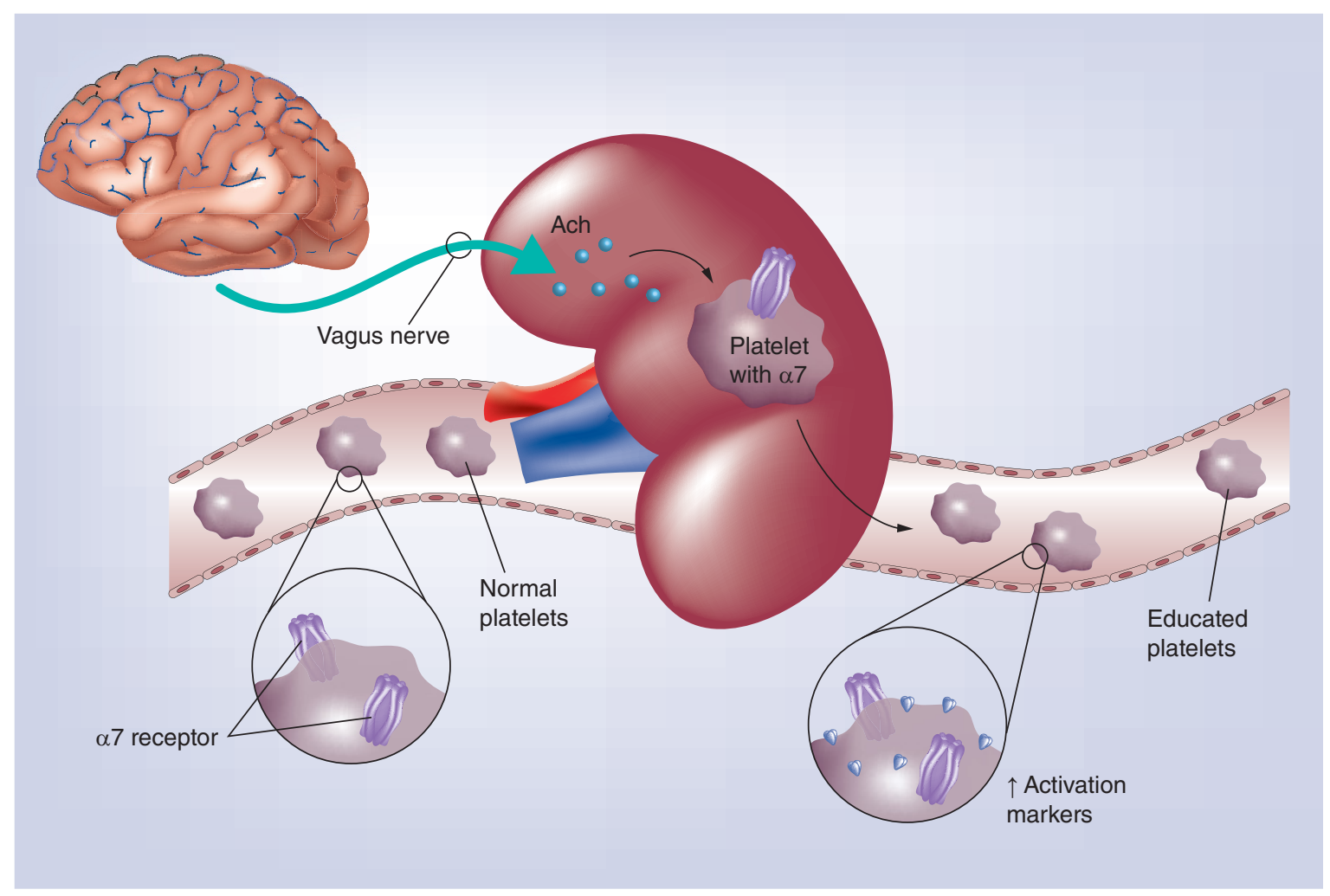

Figure 2. The Neural Tourniquet ${ }^{\mathrm{TM}}$.

Vagus nerve stimulation reduces blood loss and duration of hemorrhage in animal models of traumatic hemorrhage. Here, we propose a model whereby the CNS regulates systemic hemostasis through similar molecular, cellular and organ targets as the cholinergic anti-inflammatory pathway. This mechanism allows the brain to limit bleeding rapidly by utilizing hard-wired neural circuits, while also modulating bodily sites not innervated directly by the vagus nerve.

in hemostasis following VNS. This observation supports the hypothesis that neural based pathways act rapidly and specifically to effect change at the intended target.

While conducting these experiments, we were intrigued by how stimulating the cervical vagus nerve stops bleeding at the ear when the efferent vagus nerve does not innervate this area directly. To help explain this observation, we reexamined previous studies of the inflammatory reflex. In addition to regulating cytokine production, VNS modulates leukocyte trafficking. Electrical VNS significantly decreases accumulation of neutrophils at sites of local inflammation in mice [47]. VNS downregulates CD11b, a critical surface adhesion marker, on circulating neutrophils [48]. VNS fails to regulate leukocyte trafficking in splenectomized mice, suggesting that the brain maintains a functional synapse via the vagus nerve with circulating cells passing through the spleen [48]. This vagal connection to the spleen allows the brain to 'innervate' sites in the body without electrical signals reaching them directly. If this strategy works to control inflammation, it is possible the same neural circuitry helps modulate bleeding (Figure 2).

The molecular receptors or synapses required for the Neural Tourniquet remain unknown. Based again on the Inflammatory Reflex, the most logical candidate is the $\alpha 7$ nicotinic acetylcholine receptor subunit (7nAChR). Interestingly, a previous study identified expression of $\alpha 7 \mathrm{nAChR}$ mRNA and protein on human platelets [49]. $\alpha 7 \mathrm{nAChR}$ forms functional calcium channels and allow for calcium entry into cells. In addition to stimulating calcium uptake, cholinergic agonists enhance fibrinogen receptor activation following exposure to classical platelet agonists. Moreover, $\alpha 7 \mathrm{nAChR}$ antagonists prevent the platelet agonists from initiating platelet aggregation. Taken together, these findings suggest that $\alpha 7 \mathrm{nAChR}$ plays an important role in modulating platelet function.

Identifying the cell type(s) that receive signals from the vagus nerve to help control hemorrhage is also necessary. One significant challenge with controlling bleeding via the spleen is that putative target cells must first circulate throughout the body before reaching the site of injury. Targeting circulating neutrophils in the spleen via the vagus nerve to control inflammation appears logical because the downregulated neutrophils will not potentiate 
inflammation elsewhere as they travel in the bloodstream. In contrast, activating circulating platelets in the spleen could inadvertently precipitate potentially lethal thromboembolic complications elsewhere in the body as they travel from the spleen to the site of tissue hemorrhage.

While our experimental work up to this point focuses on bleeding in animals with normal hemostatic and coagulation pathways, it is possible to test the efficacy of the neural tourniquet in the setting of an inherited coagulopathy. Hemophilia A is an X-linked recessive disorder resulting in mild-to-severe deficiencies of Factor VIII. Affected individuals have lifelong spontaneous and prolonged bleeding after seemingly minor injuries, and require regular replacement therapy with recombinant coagulation factor products. The financial and emotional costs of chronic therapy pose a tremendous burden to patients and their families. Over time, many individuals also develop antibodies to these factor replacement preparations [50,51]. Maintaining adequate hemostatic function under these circumstances is even more problematic, and necessitates costly additional therapies. Development of a novel therapy for this disease would represent a substantial breakthrough in terms of efficacy, cost and reducing adverse effects.

Currently, we are enrolling subjects in the first pilot study to explore Neural Tourniquet function in humans. Healthy adult subjects will have baseline hematologic, platelet and coagulation pathway function analyzed followed by randomization into one of multiple electrical stimulation groups or sham controls. Based on preclinical work showing that transcutaneous VNS activates the Inflammatory Reflex, electrical VNS is performed transcutaneously [52]. We will evaluate the feasibility, tolerability and efficacy of VNS to specifically and safely augment clot formation and reduce bleeding following superficial tissue injury. Additional larger clinical trials will ultimately determine if the Neural Tourniquet can help treat traumatic hemorrhage and other bleeding disorders.

\section{Conclusion}

Bioelectronic medicine may represent the next phase in the evolution of modern medicine. While it has long been known that the nervous system monitors myriad physiological processes, it is now evident that targeted neural stimulation can regulate and even enhance these endogenous functions. Therapies such as electrical VNS are well tolerated and demonstrate efficacy where traditional pharmacological treatments have failed. Further insights into critical molecular and cellular pathways underlying systemic inflammation and hemostasis will provide more accurate and specific targets for harnessing the nervous system. Additional clinical studies are needed to ultimately demonstrate the applicability, safety and cost of bioelectronic medicine.

\section{Future perspective}

Over the next 5 to 10 years, the Neural Tourniquet has the potential to transform patient care in multiple disciplines. Noninvasive VNS delivered immediately upon arrival to the emergency room may help curtail bleeding in trauma patients. Equipping emergency medical personnel with portable stimulation devices may facilitate earlier treatment at the scene of an accident. Army medics could carry similar equipment for use on the battlefield. Patients undergoing elective surgery could receive stimulation in the holding area while waiting to go back to the operating room. Finally, children and adults with hemophilia may benefit from treatment at home utilizing miniaturized handheld devices akin to smartphones. Further technological and scientific advances may even allow bioelectronic devices to monitor endogenous clotting function and deliver an appropriate corrective stimulus in real time independent of patient or physician input.

\section{Executive summary}

- Bioelectronic medicine harnesses the nervous system to treat injury and disease through targeted manipulation of electrical neural signals.

- Current indications for bioelectronic medicine include electrical vagus nerve stimulation for the treatment of medically refractory epilepsy and depression.

- Future indications for bioelectronic medicine may include vagus nerve stimulation for the treatment of sepsis and inflammatory disorders, including rheumatoid arthritis and Crohn's disease.

- The Neural Tourniquet ${ }^{\mathrm{TM}}$ is a vagus nerve-mediated pathway that reduces traumatic bleeding by accelerating clot formation specifically at the site of injury. 


\section{Financial \& competing interests disclosure}

JM Huston is a Co-Founder and Chief Medical Advisor for Sanguistat Inc., a medical device company formed to commercialize the Neural Tourniquet ${ }^{\mathrm{TM}}$. The authors have no other relevant affiliations or financial involvement with any organization or entity with a financial interest in or financial conflict with the subject matter or materials discussed in the manuscript apart from those disclosed.

No writing assistance was utilized in the production of this manuscript.

\section{References}

1 Levi M. Management of bleeding in patients treated with direct oral anticoagulants. Crit. Care. 20, 249 (2016).

2 Roca I, Akova M, Baquero F. The global threat of antimicrobial resistance: science for intervention. New Microbes New Infect. 6, 22-29 (2015).

3 Meyer R, Patel AM, Rattana SK, Quock TP, Mody SH. Prescription opioid abuse: a literature review of the clinical and economic burden in the United States. Popul. Health Manag. 17(6), 372-387 (2014).

4 Hajar ER, Cafiero AC, Hanlon JT. Polypharmacy in elderly patients. Am. J. Geriatric Psychiatry 5(4), 345-351 (2007).

5 Altice CK, Banegas MP, Tucker-Seeley RD, Yabroff KR. Financial hardships experienced by cancer survivors: a systematic review. J. Natl Cancer Inst. 109(2), (2007).

6 Fritz JR, Huston JM. The neural tourniquet. Bioelectronic Med. 1, 25-29 (2014).

7 Pavlov VA, Tracey KJ. Neural regulation of immunity: molecular mechanisms and clinical translation. Nat. Neurosci. 20(2), 156-166 (2017).

7 Tracey KJ. The inflammatory reflex. Nature 420(6917), 853-859 (2002).

8 Ngugi AK, Kariuki SM, Bottomley C, Kleinschmidt I, Sander JW, Newton CR. Incidence of epilepsy. A systematic review and meta-analysis. Neurology 77(10), 1005-1012 (2011).

9 Tian N, Shaw EC, Zack M, Kobau R, Dykstra H, Covington TM. Cause-specific mortality among children and young adults with epilepsy: results from the U.S. National Child Death Review Case Reporting System. Epilepsy Behav. 45, 31-34 (2015).

10 Gaitatzis A, Sander JW. The long-term safety of antiepileptic drugs. CNS Drugs 27(6), 435-455 (2013).

11 Dunlop K, Hanlon CA, Downar J. Noninvasive brain stimulation treatments for addiction and major depression. Ann. NY Acad. Sci. 1294(1), 31-54 (2017).

12 Beekwilder JP, Beems T. Overview of the clinical applications of vagus nerve stimulation. J. Clin. Neurophysiol. 27, 130-138 (2010).

13 Panebianco M, Rigby A, Weston J, Marson AG. Vagus nerve stimulation for partial seizures. Cochrane Database Syst. Rev. (4), CD002896 (2015).

14 Ryvlin P, Gilliam FG, Nguyen DK et al. The long-term effect of vagus nerve stimulation on quality of life in patients with pharmacoresistant focal epilepsy: the PuLsE (Open Prospective Randomized Long-term Effectiveness) trial. Epilepsia 55(6), 893-900 (2014).

15 Grazzi L, Egeo G, Calhoun AH et al. Non-invasive vagus nerve stimulation (nVNS] as mini-prophylaxis for menstrual/menstrually related migraine: an open-label study. J. Headache Pain 17(1), 91-101 (2016).

16 Straube A, Ellrich J, Eren $\mathrm{O}$ et al. Treatment of chronic migraine with transcutaneous stimulation of the auricular branch of the vagus nerve (auricular t-VNS): a randomized, monocentric clinical trial. J. Headache Pain 16, 63-72 (2015).

17 Kinfe TM, Pintea B, Muhammad S et al. Cervical non-invasive vagus nerve stimulation (nVNS) for preventive and acute treatment of episodic and chronic migraine and migraine-associated sleep disturbance: preliminary findings from a prospective observational cohort study. J. Headache Pain 16, 101-112 (2015).

18 Watkins LR, Goehler LE, Relton JK et al. Blockade of interleukin-1 induced hyperthermia by subdiaphragmatic vagotomy: evidence for vagal mediation of immune-brain communication. Neurosci. Lett. 183(1-2), 27-31 (1995).

19 Hansen MK, Krueger JM. Subdiaphragmatic vagotomy blocks the sleep- and fever-promoting effects of interleukin-1beta. Am. J. Physiol. 273(4 Pt 2), R1246-1253 (1997).

20 Hansen MK, O'Connor KA, Goehler LE et al. The contribution of the vagus nerve in interleukin-1beta-induced fever is dependent on dose. Am. J. Physiol. Regul. Integr. Comp. Physiol. 280(4), R929-934 (2001).

21 Fleshner M, Goehler LE, Hermann J, Relton JK, Maier SF, Watkins LR. Interleukin-1 beta induced corticosterone elevation and hypothalamic NE depletion is vagally mediated. Brain Res. Bull. 37(6), 605-610 (1995).

22 Tracey KJ. The inflammatory reflex. Nature 420(6917), 853-859 (2002).

23 Tracey KJ. Physiology and immunology of the cholinergic antiinflammatory pathway. J. Clin. Invest. 117(2), 289-296 (2007).

24 Huston JM. The vagus nerve and the inflammatory reflex: wandering on a new treatment paradigm for systemic inflammation and sepsis. Surg. Infect. 13(4), 187-193 (2012). 
25 Levine YA, Koopman FA, Faltys $\mathrm{M}$ et al. Neurostimulation of the cholinergic anti-inflammatory pathway ameliorates disease in rat collagen-induced arthritis. PLoS One 9(8), e104530 (2014).

26 Niederbichler AD, Papst S, Claassen L et al. Burn-induced organ dysfunction: vagus nerve stimulation attenuates organ and serum cytokine levels. Burns 35(6), 783-789 (2009).

27 Merregnani J, Clarencon D, Vivier M et al. Anti-inflammatory effect of vagus nerve stimulation in a rat model of inflammatory bowel disease. Auton. Neurosci. 160(1-2), 82-89 (2011).

28 Mioni C, Bazzani C, Giuliani D et al. Activation of an efferent cholinergic pathway produces strong protection against myocardial ischemia/reperfusion injury in rats. Crit. Care Med. 33(11), 2621-2628 (2005).

29 van westerloo DJ, Giebelen IA, Florquin S et al. The vagus nerve and nicotinic receptors modulate experimental pancreatitis in mice. Gastroenterology 130(6), 1822-1830 (2006).

30 Ay I, Lu J, Ay H, Gregory Sorensen A. Vagus nerve stimulation reduces infarct size in rat focal cerebral ischemia. Neurosci. Lett. 459(3), $147-151$ (2009).

31 Wang H, Liao H, Ochani M et al. Cholinergic agonists inhibit HMGB1 release and improve survival in experimental sepsis. Nat. Med. 10(11), 1216-1221 (2004).

32 Borovikova LV, Ivanova S, Zhang M et al. Vagus nerve stimulation attenuates the systemic inflammatory response to endotoxin. Nature 405(6785), 458-462 (2000).

33 Huston JM, Ochani M, Rosas-Ballina M et al. Splenectomy inactivates the cholinergic antiinflammatory pathway during lethal endotoxemia and polymicrobial sepsis. J. Exp. Med. 203(7), 1623-1628 (2006).

34 Rosas-Ballina M, Ochani M, Parrish WR et al. Splenic nerve is required for cholinergic antiinflammatory pathway control of TNF in endotoxemia. Proc. Natl Acad. Sci. USA 105(31), 11008-11013 (2008).

35 Rosas-Ballina M, Olofsson PS, Ochani M et al. Acetylcholine-synthesizing T cells relay neural signals in a vagus nerve circuit. Science 334(6052), 98-101 (2011).

36 Wang $\mathrm{H}, \mathrm{Yu} \mathrm{M}$, Ochani $\mathrm{M}$ et al. Nicotinic acetylcholine receptor alpha7 subunit is an essential regulator of inflammation. Nature 421(6921), 384-388 (2003).

37 Bonaz B, Sinniger V, Hoffmann D et al. Chronic vagus nerve stimulation in Crohn's disease: a 6-month follow-up study. Neurogastroenterol. Motil. 28, 948-953 (2016).

38 Koopman FA, Chavan SS, Miljko S et al. Vagus nerve stimulation inhibits cytokine production and attenuates disease severity in rheumatoid arthritis. Proc. Natl Acad. Sci. USA 113(29), 8284-8289 (2016).

39 Gomez-Barrena E, Ortega-Andreu M, Padilla-Eguiluz NG et al. Topical intra-articular compared with intravenous tranexamic acid to reduce blood loss in primary total knee replacement: a double-blind, randomized, controlled, noninferiority clinical trial. J. Bone Joint Surg. Am. 96(23), 1937-1944 (2014).

40 Maniar RN, Kumar G, Singhi T et al. Most effective regimen of tranexamic acid in knee arthroplasty: a prospective randomized controlled study in 240 patients. Clin. Orthop. Relat. Res. 470(9), 2605-2612 (2012).

41 Classen DC, Evans RS, Pestotnik SL et al. The timing of prophylactic administration of antibiotics and the risk of surgical-wound infection. N. Engl. J. Med. 326(5), 281-286 (1992).

42 Mackeen AD, Packard RE, Ota E et al. Timing of intravenous prophylactic antibiotics for preventing postpartum infectious morbidity in women undergoing cesarean delivery (Review). Cochrane Database of Systematic Reviews (12), 1-50 (2014).

43 Kiil J, Kiil J, Axelsen F et al. Prophylaxis against postoperative pulmonary embolism and deep-vein thrombosis by low-dose heparin. Lancet 1(8074), 1115-1116 (1978).

44 Henke PK, Arya S, Pannucci C et al. Procedure-specific thromboembolism prophylaxis: a paradigm from colectomy surgery. Surgery 152(4), 528-536 (2012).

45 Pidcoke HF, Aden JK, Mora AG et al. Ten-year analysis of transfusion in Operation Iraqi Freedom and Operation Enduring Freedom: increased plasma and platelet use correlates with improved survival. J. Trauma Acute Care Surg. 73(6 Suppl. 5), S445-452 (2012).

46 Czura CJ, Schultz A, Kaipel M et al. Vagus nerve stimulation regulates hemostasis in swine. Shock 33(6), 608-613 (2010).

47 Saeed RW, Varma S, Peng-Nemeroff T et al. Cholinergic stimulation blocks endothelial cell activation and leukocyte recruitment during inflammation. J. Exp. Med. 201(7), 1113-1123 (2005).

48 Huston JM, Rosas-Ballina M, Xue X et al. Cholinergic neural signals to the spleen down-regulate leukocyte trafficking via CD11b. J. Immunol.183(1), 552-559 (2009).

49 Schedel A, Thornton S, Schloss P et al. Human platelets express functional alpha7-nicotinic acetylcholine receptors. Arterioscler. Thromb. Vasc. Biol. 31(4), 928-934 (2011).

50 Gouw SC, van der Bom JG, Ljung R et al. Factor VIII products and inhibitor development in severe hemophilia A. N. Engl. J. Med. 368(3), 231-239 (2013).

51 Peyvandi F, Mannucci PM, Garagiola I et al. A randomized trial of factor VIII and neutralizing antibodies in hemophilia A. N. Engl. J. Med. 374(21), 2054-2064 (2016). 
Review Huston \& Fritz

52 Huston JM, Gallowitsch-Puerta M, Ochani M et al. Transcutaneous vagus nerve stimulation reduces serum high mobility group box 1 levels and improves survival in murine sepsis. Crit. Care Med. 35(12), 2762-2768 (2007). 